\title{
The nullspace method - a unifying paradigm to fault detection
}

\author{
A. Varga
}

\begin{abstract}
The nullspace method is a powerful framework to solve the synthesis problem of fault detection filters in the most general setting. It is also well suited to address the least order synthesis problem. In the same time, the nullspace method represents an unifying paradigm for several methods, because popular approaches like parity space or observer based methods can be interpreted as special classes of nullspace method. The main differences among different methods lie in the numerical properties of the underlying computational algorithms.
\end{abstract}

\section{The FAUlT DeTECTION PROBLEM}

Consider additive fault models described by input-output representations of the form

$$
\mathbf{y}(\lambda)=G_{u}(\lambda) \mathbf{u}(\lambda)+G_{d}(\lambda) \mathbf{d}(\lambda)+G_{f}(\lambda) \mathbf{f}(\lambda),
$$

where $\mathbf{y}(\lambda), \mathbf{u}(\lambda), \mathbf{d}(\lambda)$, and $\mathbf{f}(\lambda)$ are Laplace- or Ztransformed vectors of the the $p$-dimensional system output vector $y(t), m_{u}$-dimensional control input vector $u(t), m_{d^{-}}$ dimensional disturbance vector $d(t)$, and $m_{f}$-dimensional fault vector $f(t)$, respectively, and where $G_{u}(\lambda), G_{d}(\lambda)$ and $G_{f}(\lambda)$ are the transfer-function matrices (TFMs) from the control inputs to outputs, disturbance inputs to outputs, and fault inputs to outputs, respectively. According to the system type, the frequency variable $\lambda$ is either $s$, the complex variable in the Laplace-transform in the case of a continuoustime system, or $z$, the complex variable in the Z-transform in the case of a discrete-time system. For most of practical applications, the TFMs $G_{u}(\lambda), G_{d}(\lambda)$ and $G_{f}(\lambda)$ are proper rational matrices (i.e., only with finite poles). However, for complete generality of our problem formulation, we will allow that these TFMs are general non-proper rational matrices for which we will not a priori assume any further properties (e.g., stability, full rank).

A linear residual generator (or fault detection filter) processes the measurable system outputs $y(t)$ and control inputs $u(t)$ and generates the residual signals $r(t)$ which serve for decision making on the presence or absence of faults. The input-output form of this filter is

$$
\mathbf{r}(\lambda)=Q(\lambda)\left[\begin{array}{l}
\mathbf{y}(\lambda) \\
\mathbf{u}(\lambda)
\end{array}\right]
$$

where $Q(\lambda)$ is the TFM of the filter. For a physically realizable filter, $Q(\lambda)$ must be proper and stable (i.e., only with poles having negative real parts for a continuous-time system or magnitudes less than one for a discrete-time system). The (dynamic) order of $Q(\lambda)$ (also known as McMillan degree)

This work was supported via the ONERA-DLR Project IMMUNE: Intelligent Monitoring and Management of Unexpected Events.

A. Varga is with the German Aerospace Center, DLR - Oberpfaffenhofen, Institute of Robotics and Mechatronics, D-82234 Wessling, Germany. Andras.Vargaedlr.de is the dimension of the state vector of a minimal state-space realization of $Q(\lambda)$. The dimension $q$ of the residual vector $r(t)$ depends on the fault detection problem to be solved, and can be either given or determined during the filter synthesis. For example, for the detection of faults a single residual is sufficient, but for a better balance of detection sensitivities of faults a set of residuals could be advantageous.

The residual signal $r(t)$ in (2) generally depends via the system outputs $y(t)$ of all system inputs $u(t), d(t)$ and $f(t)$. The residual generation system, obtained by replacing in (2) $\mathbf{y}(\lambda)$ by its expression from (1), is given by

$$
\mathbf{r}(\lambda)=R_{f}(\lambda) \mathbf{f}(\lambda)+R_{u}(\lambda) \mathbf{d}(\lambda)+R_{d}(\lambda) \mathbf{u}(\lambda)
$$

where

$$
\left[R_{f}(\lambda)\left|R_{u}(\lambda)\right| R_{d}(\lambda)\right]:=Q(\lambda)\left[\begin{array}{c|c|c}
G_{f}(\lambda) & G_{u}(\lambda) & G_{d}(\lambda) \\
0 & I_{m_{u}} & 0
\end{array}\right]
$$

For a successfully designed filter $Q(\lambda)$, the corresponding residual generation system is proper and stable and achieves specific fault detection requirements (e.g., decoupling of control and disturbance inputs from the residuals).

The fault detection problem (FDP) can be formulated as follows: Determine a physically realizable linear residual generator filter having the general form (2) such that for all $d(t)$ and $u(t)$ we have:

(i) $r(t)=0$ when $f(t)=0$;

(ii) $r(t) \neq 0$ when any $f_{j}(t) \neq 0$, for $j=1, \ldots, m_{f}$;

(iii) $r(t)$ is asymptotically bounded.

Besides the above requirements it is often required for practical use that the TFM of the detector $Q(\lambda)$ has the least possible McMillan degree.

Let $G_{f_{i}}(\lambda)$ be the $i$-th column of $G_{f}(\lambda)$. A necessary and sufficient condition for the existence of a solution is the following one [1], [2]:

Theorem 1: For the system (1) the FDP is solvable if and only if

$$
\operatorname{rank}\left[G_{d}(\lambda) G_{f_{i}}(\lambda)\right]>\operatorname{rank} G_{d}(\lambda), \quad i=1, \ldots, m_{f}
$$

The requirements $(i)$ and (ii) of the FDP can be easily transcribed into equivalent algebraic conditions. The decoupling condition $(i)$ requires $R_{u}(\lambda)=0$ and $R_{d}(\lambda)=0$ and thus is equivalent to

$$
Q(\lambda) G(\lambda)=0
$$

where

$$
G(\lambda)=\left[\begin{array}{cc}
G_{u}(\lambda) & G_{d}(\lambda) \\
I_{m_{u}} & 0
\end{array}\right] .
$$

The detectability condition $(i i)$ is equivalent to

$$
R_{f_{i}}(\lambda) \neq 0, \quad i=1, \ldots, m_{f},
$$


where $R_{f_{i}}(\lambda)$ is the $i$-th column of $R_{f}(\lambda)$. A detector $Q(\lambda)$ satisfying (5) and (7) is called admissible. The boundedness condition (iii) of residual signals requires that both $Q(\lambda)$ and $R_{f}(\lambda)$ are proper and stable TFMs.

From (5) it appears that $Q(\lambda)$ is a left annihilator of $G(\lambda)$, thus one possibility to determine $Q(\lambda)$ is to compute first a minimal basis $N_{l}(\lambda)$ for the left nullspace of $G(\lambda)$, and then to build a proper and stable detector as

$$
Q(\lambda)=W(\lambda) N_{l}(\lambda),
$$

where $W(\lambda)$ is a suitable rational matrix which additionally ensures that conditions (7) and the stability requirements are fulfilled. The expression (8) represents a parametrization of all possible fault detection filters and is the basis of the nullspace methods for the synthesis of residual generators. Details of the computational aspects of this method are provided in the next section.

For computational purposes, we exclusively employ a descriptor state space realization of (1)

$$
\begin{aligned}
E \lambda x(t) & =A x(t)+B_{u} u(t)+B_{d} d(t)+B_{f} f(t) \\
y(t) & =C x(t)+D_{u} u(t)+D_{d} d(t)+D_{f} f(t)
\end{aligned},
$$

where $x(t)$ is the $n$-dimensional state vector, and $\lambda x(t)=$ $\dot{x}(t)$ or $\lambda x(t)=x(t+1)$ depending on the type of the system, continuous or discrete, respectively. In general we can assume that the realization (9) is regular and irreducible, that is, $\operatorname{det}(A-\lambda E) \not \equiv 0$, the pair $(A-\lambda E, C)$ is observable and the pair $\left(A-\lambda E,\left[\begin{array}{lll}B_{u} & B_{d} & B_{f}\end{array}\right]\right)$ is controllable. For a proper system we can always assume a realization with $E$ invertible, or even a standard state space realization with $E=I_{n}$.

Let $B_{f_{i}}$ and $D_{f_{i}}$ denote the $i$-th columns of matrices $B_{f}$ and $D_{f}$, respectively. An equivalent formulation of the necessary and sufficient conditions in Theorem 1 has been derived in [3], [4]:

Theorem 2: For the system (1) the FDP is solvable if and only if for $i=1, \ldots, m_{f}$ we have

$$
\operatorname{rank}\left[\begin{array}{ccc}
A-\lambda E & B_{d} & B_{f_{i}} \\
C & D_{d} & D_{f_{i}}
\end{array}\right]>\operatorname{rank}\left[\begin{array}{cc}
A-\lambda E & B_{d} \\
C & D_{d}
\end{array}\right]
$$

In this paper we show that the nullspace method provides a unifying paradigm for the main synthesis methods of fault detection filters. Popular methods as the parity space or diagnostic observer based techniques can be interpreted as special cases of the nullspace method. The equivalence between these two methods has been already investigated (see for example [5], [6] and references cited therein). However, when comparing different approaches, besides the strict mathematical equivalence, also other aspects must play a role, as for example, the ability to solve the FDP in the most general setting, the quality of underlying numerics, or even the ability to address the important aspect of synthesizing least order fault detection filters. From all these viewpoints, the existing methods by no means can be considered equivalent. In the light of the importance of solving FDPs in various related problems (e.g., stochastic fault detection, exact or optimal fault detection and isolation), a critical discussion of these aspects, never done before, is timely to orient the choice of best techniques and software tools.

\section{NullspaCE METHOD}

The nullspace method relies on fulfilling conditions (5) and (7) and is conceptually very simple, consisting of three main steps:

1) Compute a left nullspace basis $N_{l}(\lambda)$ of $G(\lambda)$ defined in (6). Exit if $N_{l}(\lambda)$ is not admissible (no solution).

2) Compute a rational matrix $W(\lambda)$ such that $\widetilde{Q}(\lambda):=$ $W(\lambda) N_{l}(\lambda)$ is admissible and has the least order.

3) Compute a suitable rational matrix $M(\lambda)$ such that $Q(\lambda):=M(\lambda) \widetilde{Q}(\lambda)$ and $R_{f}(\lambda)$ are proper and stable.

The nullspace method has been formally introduced in [7], where the employed basis $N_{l}(\lambda)$ was a minimal polynomial basis. The polynomial basis method has been recently extended to address the more general case of nonproper models [8]. The main advantage of this approach is that the least order design aspect is naturally present in the formulation of the method. The choice of suitable $W(\lambda)$ and $M(\lambda)$ to ensure stability and properness is straightforward and can be done in one step. The main limitation of the polynomial basis method is of numerical nature. By computing explicitly such a basis, the method potentially suffers of lack of numerical reliability in the case of higher order systems. More details in this respect can be found in [9].

To improve the numerical properties of the nullspace method, the polynomial nullspace basis based method has been extended to rational nullspace bases in [9], [10], [11]. In what follows we present some details of the underlying computational aspects for each step of the above procedure.

Step 1): The computation of a rational left nullspace basis $N_{l}(\lambda)$ is performed using a matrix pencil method based on the state space representation of the $\left(p+m_{u}\right) \times\left(m_{u}+m_{d}\right)$ rational matrix

$$
G(\lambda)=\left[\begin{array}{c|cc}
A-\lambda E & B_{u} & B_{d} \\
\hline C & D_{u} & D_{d} \\
0 & I_{m_{u}} & 0
\end{array}\right] .
$$

The computational method described in [9] exploits the simple fact that $N_{l}(\lambda)$ is a left nullspace basis of $G(\lambda)$ if and only if for a suitable $M_{l}(\lambda)$

$$
Y_{l}(\lambda):=\left[M_{l}(\lambda) \mid N_{l}(\lambda)\right]
$$

is a left nullspace basis of the correspondingly partitioned system matrix

$$
S(\lambda)=\left[\begin{array}{ccc}
A-\lambda E & B_{u} & B_{d} \\
\hline C & D_{u} & D_{d} \\
0 & I_{m_{u}} & 0
\end{array}\right] .
$$

Thus, to compute $N_{l}(\lambda)$ we determine first a left nullspace basis $Y_{l}(\lambda)$ for $S(\lambda)$ and then $N_{l}(\lambda)$ simply results as

$$
N_{l}(\lambda)=Y_{l}(\lambda)\left[\begin{array}{c}
0 \\
I_{p+m_{u}}
\end{array}\right]
$$

To compute $Y_{l}(\lambda)$, we employ linear matrix pencil reduction algorithms based on orthogonal transformations. Let $U$ 
and $V$ be orthogonal matrices (for instance, determined by using the algorithms of [12], [13]) such that the transformed pencil $\widetilde{S}(\lambda):=U S(\lambda) V$ is in the Kronecker-like staircase form

$$
\widetilde{S}(\lambda)=\left[\begin{array}{cc}
A_{r}-\lambda E_{r} & A_{r, l}-\lambda E_{r, l} \\
\hline 0 & A_{l}-\lambda E_{l} \\
\hline 0 & C_{l}
\end{array}\right]
$$

where the descriptor pair $\left(A_{l}-\lambda E_{l}, C_{l}\right)$ is observable, $E_{l}$ is non-singular, and $A_{r}-\lambda E_{r}$ has full row rank excepting possibly a finite set of values of $\lambda$ (i.e, the invariant zeros of $S(\lambda)$ ). We can choose a proper left nullspace $\widetilde{Y}_{l}(\lambda)$ of $\widetilde{S}(\lambda)$ in the form

$$
\widetilde{Y}_{l}(\lambda)=\left[0\left|C_{l}\left(\lambda E_{l}-A_{l}\right)^{-1}\right| I\right] .
$$

Then, a left nullspace of $S(\lambda)$ is

$$
Y_{l}(\lambda)=\widetilde{Y}_{l}(\lambda) Q\left[\begin{array}{c}
0 \\
I_{p+m_{u}}
\end{array}\right]
$$

If we partition row-wise

$$
Q\left[\begin{array}{c}
0 \\
I_{p+m_{u}}
\end{array}\right]=\left[\begin{array}{c}
B_{r, l} \\
B_{l} \\
D_{l}
\end{array}\right]
$$

to comply with the column partitioning of $\widetilde{Y}_{l}(\lambda)$ in (14), we obtain a minimal order proper left nullspace $N_{l}(\lambda)$ of $G(\lambda)$ with the descriptor realization

$$
N_{l}(\lambda)=\left[\begin{array}{c|c}
A_{l}-\lambda E_{l} & B_{l} \\
\hline C_{l} & D_{l}
\end{array}\right]
$$

To obtain this representation of the nullspace basis, we performed exclusively orthogonal transformations on the system matrices and the algorithm to compute the nullspace basis is provably numerically backward stable.

Step 2): The computation of least order detectors can be addressed by employing $W(\lambda)$ of the form

$$
W(\lambda)=\left[\begin{array}{c|c}
A_{l}+K C_{l}-\lambda E_{l} & K \\
\hline H C_{l} & H
\end{array}\right]
$$

where $H$ and $F$ are determined such that

$$
W(\lambda) N_{l}(\lambda)=\left[\begin{array}{c|c}
A_{l}+K C_{l}-\lambda E_{l} & B_{l}+K D_{l} \\
\hline H C_{l} & H D_{l}
\end{array}\right]
$$

has a maximum number of canceled (unobservable) poles and the detector $\widetilde{Q}(\lambda)=W(\lambda) N_{l}(\lambda)$ is admissible. For a given $H$, the computation of the corresponding $K$ can be done by solving a minimum dynamic cover problem [10], for which an efficient computational algorithm has been proposed in [14]. Although this algorithm partly relies on non-orthogonal similarity transformations, still its numerically reliability is guaranteed, because any loss of numerical stability can be easily detected. The choice of appropriate $H$ is described in [10] for scalar output detectors, and exploits the resulting observability staircase form of the pair $\left(A_{l}-\lambda E_{l}, C_{l}\right)$, which also contains the complete information on the achievable orders. The selection approach of $H$ described in [10] can be readily extended to detectors with arbitrary number of outputs.
An optimal choice of $H$ can substantially improve the sensitivity of detectors to several faults. Ideally, the residual should exhibit the same sensitivity to each fault. However, in practice, such a goal can be seldom achieved, and therefore it is a good idea to try reduce the gap between the largest and smallest gains among the columns of $R_{f}(\lambda)$. A suitable sensitivity condition of a residual to individual faults has been introduced in [15] as the largest relative column gain

$$
\xi:=\max _{j}\left\|R_{f_{j}}(\lambda)\right\|_{\infty} / \min _{j}\left\|R_{f_{j}}(\lambda)\right\|_{\infty}
$$

By choosing $H$ to minimize $\xi$, the gap in the column gains is automatically minimized.

Step 3): The computation of $M(\lambda)$ is necessary to ensure a desired dynamics of the detector $Q(\lambda)$ and of the corresponding $R_{f}(\lambda)$. This can be achieved by using standard left coprime factorization techniques [16] using a square, invertible, proper and stable $M(\lambda)$. A more sophisticated numerically reliable procedure to determine simultaneously $M(\lambda)$ and $Q(\lambda)=M(\lambda) \widetilde{Q}(\lambda)$ is based on a sequential pole assignment based approach [17].

\section{PARITY SPACE METHOD}

The parity relations based approach has been pioneered in [18], [19] for standard discrete-time systems and since then widely discussed in the literature. An extension to standard continuous-time systems has been discussed in [5], while an extension to discrete-time descriptor systems has been proposed in [20].

The basic approach consists in using a pseudo-residual computed as

$$
\widetilde{r}(t)=H_{k}\left(\bar{y}(t)-S_{u, k} \bar{u}(t)\right),
$$

where $H_{k}$ is a so-called parity matrix with $p(k+1)$ columns chosen in a special way (see below),

$$
S_{u, k}:=\left[\begin{array}{cccc}
D_{u} & 0 & \cdots & 0 \\
C B_{u} & D_{u} & \ddots & \vdots \\
\vdots & \ddots & \ddots & 0 \\
C A^{k-1} B_{u} & \cdots & C B_{u} & D_{u}
\end{array}\right]
$$

and, depending of the system type

$$
\begin{aligned}
& \bar{y}^{T}(t)=\left[\begin{array}{llll}
y^{T}(t) & \left(\frac{d y(t)}{d t}\right)^{T} & \cdots & \left(\frac{d^{k} y(t)}{d t^{k}}\right)^{T}
\end{array}\right] \\
& \bar{u}^{T}(t)=\left[\begin{array}{llll}
u^{T}(t) & \left(\frac{d u(t)}{d t}\right)^{T} & \cdots & \left(\frac{d^{k} u(t)}{d t^{k}}\right)^{T}
\end{array}\right]
\end{aligned}
$$

for a continuous-time system, or

$$
\begin{aligned}
& \bar{y}^{T}(t)=\left[\begin{array}{llll}
y^{T}(t) & y^{T}(t+1) & \cdots & y^{T}(t+k)
\end{array}\right] \\
& \bar{u}^{T}(t)=\left[\begin{array}{llll}
u^{T}(t) & u^{T}(t+1) & \cdots & u^{T}(t+k)
\end{array}\right]
\end{aligned}
$$

for a discrete-time system.

The matrix $H_{k}$ is determined such that

$$
H_{k}\left[\begin{array}{ll}
S_{0, k} & S_{d, k}
\end{array}\right]=0, \quad H_{k} S_{f_{i}, k} \neq 0, \quad i=1, \ldots, m_{f}
$$


where $S_{d, k}$ and $S_{f_{i}, k}$ are matrices of the form (18) corresponding to the disturbance input $d(t)$ and fault input $f_{i}(t)$, while $S_{0, k}$ is the matrix

$$
S_{0, k}=\left[\begin{array}{c}
C \\
C A \\
\vdots \\
C A^{k}
\end{array}\right]
$$

The necessary and sufficient conditions for the existence of a solution are equivalent to those of Theorem 1 [21].

Theorem 3: For the system (1) the FDP is solvable if and only if for $k \geq n$ we have for $i=1, \ldots, m_{f}$

$$
\operatorname{rank}\left[\begin{array}{lll}
S_{0, k} & S_{d, k} & S_{f_{i}, k}
\end{array}\right]>\operatorname{rank}\left[\begin{array}{ll}
S_{0, k} & S_{d, k}
\end{array}\right]
$$

The Laplace- or Z-transformed pseudo-residual $\widetilde{\mathbf{r}}(\lambda)$ can be expressed as

$$
\widetilde{\mathbf{r}}(\lambda)=\widetilde{Q}(\lambda)\left[\begin{array}{l}
\mathbf{y}(\lambda) \\
\mathbf{u}(\lambda)
\end{array}\right],
$$

where $\widetilde{Q}(\lambda)$ is a polynomial matrix with maximal row degree $k$ which can be obtained from (17) by straightforward computations. It can be shown that $\widetilde{Q}(\lambda) G(\lambda)=0$, and thus $\widetilde{Q}(\lambda)$ is a left annihilator of $G(\lambda)$ in (6). For the implementation of the residual, an additional filtering is still necessary to be added to ensure the physical realizability of the detector. A proper and stable residual filter can be determined in the form

$$
Q(\lambda)=M(\lambda) \widetilde{Q}(\lambda),
$$

where $M(\lambda)$ can be chosen a diagonal rational matrix $M(\lambda)=I / \psi(\lambda)$, with the polynomial $\psi(\lambda)$ of degree $k$ which specifies the filter dynamics. In the discrete-time case, a physically realizable dead-beat filter can be directly obtained if the alternatively definition is used for $\bar{y}(t)$ and $\bar{u}(t)$

$$
\begin{aligned}
& \bar{y}^{T}(t)=\left[\begin{array}{llll}
y^{T}(t-k) & y^{T}(t-k+1) & \cdots & y^{T}(t)
\end{array}\right] \\
& \bar{u}^{T}(t)=\left[\begin{array}{llll}
u^{T}(t-k) & u^{T}(t-k+1) & \cdots & u^{T}(t)
\end{array}\right]
\end{aligned}
$$

This corresponds to choosing $\psi(z)=z^{k}$.

The value of $k$, representing the number of differentiations in the continuous-time or the size of delays in the discretetime, determines thus the order of a proper residual generation filter which can be derived from the residual generation formula (17). For a least order design, $k$ must be chosen as the smallest value for which the solvability conditions in (19) are fulfilled. This involves a search for increasing values of $k$. Determining the least value of $k$, has been apparently addressed for the first time in [22], in the case of absence of disturbance inputs. This aspect is further discussed in [23].

Regarding the computational aspects of the parity space approach, the matrix $H_{k}$ can be easily computed from a rank-revealing QR-decomposition

$$
\left[\begin{array}{ll}
S_{0, k} & S_{d, k}
\end{array}\right]=U\left[\begin{array}{l}
Y \\
0
\end{array}\right]
$$

where $Y$ is a full row rank matrix and $U$ is an orthogonal matrix. If we partition $U=\left[\begin{array}{ll}U_{1} & U_{2}\end{array}\right]$ according to the row partitioning of the rightmost matrix above, then $H_{k}$ can be chosen as $U_{2}^{T}$, or any selection of the rows of $U_{2}^{T}$, or just a linear combination of these rows.

The parity space approach appears to be very simple and also allows to search systematically for a solution of least order. However, the computation of the parity matrix $H_{k}$ requires to form explicitly matrix powers to build the underlying matrices in (19). Matrix powers also appear in the expression of the pseudo-residual and thus when building $\widetilde{Q}(\lambda)$. Even for small state dimensions, forming matrix powers is numerically not recommendable, because it can lead to severe accuracy loss and can falsify the rank information [24]. Therefore, the parity space approach in this simple form can not be seen as a satisfactory numerical method to serve as basis for robust numerical software implementation. The suggested "enhancement" (see for example [23]) to use the Luenberger observability canonical form of the pair $(A, C)$ in order to alleviate the problematic of raising $A$ to powers, leads to other numerical difficulties because of potentially extreme ill-conditioning of the similarity transformations involved in computing such canonical forms.

\section{OBSERVER BASED METHODS}

Observer based methods proposed for solving the FDP use so-called diagnostic observers to generate residual signals. For simplicity, we only consider standard state space systems for which most of synthesis methods have been developed. In the most general setting, we can try to determine a residual generator $Q(\lambda)$ with a state space realization of order $k$ of the general form

$$
\begin{aligned}
\lambda z(t) & =F z(t)+K_{u} u(t)+K_{y} y(t) \\
r(t) & =W z(t)+H_{u} u(t)+H_{y} y(t)
\end{aligned}
$$

and try to determine the involved matrices by solving the so-called Luenberger equations [25]

$$
\begin{array}{ll}
\text { (a) } & T A-F T=K_{y} C \\
(b) & K_{u}=T B_{u}-K_{y} D_{u} \\
\text { (c) } & W T+H_{y} C=0 \\
(d) & H_{u}=-H_{y} D_{u} \\
(e) & T B_{d}-K_{y} D_{d}=0 \\
(f) & H_{y} D_{d}=0
\end{array}
$$

where $F$ is a stable matrix and $T$ is $k \times n$ matrix to be chosen. If we denote by $e(t)=z(t)-T x(t)$, then, provided the above conditions are fulfilled, the residual generator can be alternatively expressed in the form

$$
\begin{aligned}
\lambda e(t) & =F e(t)+\left(K_{y} D_{f}-T B_{f}\right) f(t) \\
r(t) & =W e(t)+H_{y} D_{f} f(t)
\end{aligned}
$$

The conditions $(a)-(f)$ express algebraically the state space equivalents for

$$
Q(\lambda)\left[\begin{array}{cc}
G_{u}(\lambda) & G_{d}(\lambda) \\
I_{m_{u}} & 0
\end{array}\right]=0
$$


and therefore $Q(\lambda)$ is a left annihilator of $G(\lambda)$ in (6). Further, (23) is the resulting realization of

$$
R_{f}(\lambda)=W(\lambda I-F)^{-1}\left(K_{y} D_{f}-T B_{f}\right)+H_{y} D_{f}
$$

and to fulfill the FDP solvability conditions we additionally need to fulfil (7). Assuming the pair $(F, W)$ is observable, condition (7) requires

$$
\left[\begin{array}{c}
K_{y} D_{f_{i}}-T B_{f_{i}} \\
H_{y} D_{f_{i}}
\end{array}\right] \neq 0, i=1, \ldots, m_{f}
$$

It follows that the fault detection observer based approach can be reinterpreted as a special instance of the nullspace method, where a residual generator of fixed order is directly determined. The main computational task is to solve the Luenberger equations $(a)-(f)$. In the fault detection literature a great deal of approaches have been proposed for this purpose. In what follows, we comment shortly the main classes of approaches.

1) Luenberger-type fault detectors : The observer based approach has been pioneered by Beard and Jones [26], [27] to solve fault isolation problems for standard state space systems using full order Luenberger-type observers. As residual generator, the following Luenberger-type output signal observer is used

$$
\begin{aligned}
\lambda \widehat{x}(t) & =A \widehat{x}(t)+B_{u} u(t)-K\left(y(t)-C \widehat{x}(t)-D_{u} u(t)\right) \\
r(t) & =H\left(y(t)-C \widehat{x}(t)-D_{u} u(t)\right)
\end{aligned}
$$

where the matrices $K$ and $H$ are chosen to achieve, via a stable observer, the conditions $(i)-(i i i)$ of the FDP. This observer corresponds to take $T=I_{n}, K_{y}=-K$ and $H_{y}=$ $H$ in the Luenberger equations (22).

If we define $e(t)=x(t)-\widehat{x}(t)$, then the residual dynamics can be equivalently expressed as

$$
\begin{aligned}
\lambda e(t) & =(A+K C) e(t)+\left(B_{f}+K D_{f}\right) f(t)+\left(B_{d}+K D_{d}\right) d(t) \\
r(t) & =H C e(t)+H D_{f} f(t)+H D_{d} d(t)
\end{aligned}
$$

To fulfill requirements $(i)$ and $(i i)$ of the FDP, the TFM $R_{d}(\lambda)$ from $d$ to $r$ must be zero, i.e.

$$
R_{d}(\lambda)=H C(\lambda I-A-K C)^{-1}\left(B_{d}+K D_{d}\right)+H D_{d}=0
$$

and each TFM $R_{f_{i}}(\lambda)$ from the fault input $f_{i}$ to $r$ for $i=$ $1, \ldots, m_{f}$ must be non-zero

$$
R_{f_{i}}(\lambda)=H C(\lambda I-A-K C)^{-1}\left(B_{f_{i}}+K D_{f_{i}}\right)+H D_{f_{i}} \neq 0
$$

The solution of this problem has been addressed by various authors using different methods, as for example, eigenstructure assignment [28], [29] or geometric methods [22] (only for the case $D_{d}=0$ and $D_{f}=0$ ). The main difficulty with this approach is that the conditions for the solvability of the FDP (see for example Theorems 1 or 2) and the conditions for the existence of an observer of the form (24) which ensures (25) and (26) (see [30, Theorems $7.6 \&$ 7.9]), are different. Thus there exists cases when although the FDP is solvable, however no observer of the form (24) can be used as residual generator. Moreover, it is also possible that although both the decoupling and detectability conditions (25) and (26) can be satisfied, however, due to unstable fixed modes, the resulting detector is also unstable [30]. Therefore, the restriction to use full-order observers is a rather severe constraint, significantly limiting the class of problems which can be solved.

2) Unknown-input observer: A closely related approach is based on the so-called unknown-input observer (UIO) (see [29] and references cited therein). An UIO aims to estimate the state of the system (9) such that for $f=0$, the estimation error $e(t)=x(t)-\widehat{x}(t)$ is independent of the disturbance inputs $d(t)$. A residual is formed as the difference between the measured and the estimated outputs (usually $D_{d}=0$ is assumed). A more advanced approach tries to estimate a linear combination $T x(t)$ of the state by fully decoupling the disturbance inputs. As pointed out in [31], [25], the main problem with this approach is that the existence conditions of UIOs differ from those of a fault detection observer. An example in [25] illustrates that while the FDP may have a solution, no solution can be obtained by using an UIO.

3) Direct solution approach: The direct design of fault detection observers has been considered in several works [31], [25], [3] (to cite a few of them). As was noted in [25], the main computational problem is the construction of a suitable $T$ matrix. The approach of [31] relies on the Kronecker canonical form of a certain matrix pencil and allows to construct $T$ using the elements of this form. Looking to the details of this approach, the computation of $T$ is solely done in terms of the left Kronecker structure, and therefore this method can be seen as a precursor of the nullspace methods. The work of [25] improves the approach of [31] by eliminating the need to compute the Kronecker form. Instead, by using a series of orthogonal transformations (strongly resembling to the structure algorithm of Silverman [32]), the disturbance inputs are finally decoupled from the original system and, simultaneously, the fault detectability conditions can be checked. Finally, a suitable $T$ is built and the solution is found by designing a standard observer for a reduced order system. The limitation of this approach to consider only the case without direct feedthrough terms from the fault inputs has been removed in [3] and the method has been latter extended to descriptor systems in [4]. This method can be easily reinterpreted as a rational nullspace basis method (actually the first one proposed in the literature). The suggested numerical approach employs an alternative Kronecker-like form and, excepting some nonessential details, it is basically equivalent with Step 1) of the nullspace approach to determine $N_{l}(\lambda)$ presented in Section II. The final design of a full order observer corresponds to the left coprime factorization performed at Step 3) of the nullspace method. However, the least order design aspect at Step 2) is not addressed at all in [3], [4].

4) Indirect solution approach: This approach combines the parity space and the observer design methods [21]. The main advantage of this combination method is its ability to address the least order design problem as well. The parity approach is used to determine the (least) order and provides a parity matrix to be used to choose the observer matrices in a particular observability canonical form. The existing 
free parameters are used to assign an arbitrary dynamics of the observer. The main weakness of this approach is the numerical computation of the parity matrix, which involves forming matrix products, and thus, is potentially numerically unstable.

\section{CONCLUSIONS}

The nullspace method provides a unifying paradigm for most of existing synthesis methods of residual generators which solves the FDP, including the parity space and diagnosis observer based approaches. In spite of this common feature, the existing methods significantly differs in their ability to address different aspects of the problem solving (e.g., finding least order solutions) or in the underlying computational algorithms. The parity space based methods are not recommendable as computational methods due to the need to form matrix powers, which almost always lead to numerical instability. The application of diagnosis observer based approaches is often only possible under strong technical assumptions or for particular model types (e.g., no feedthrough terms). The direct synthesis approach in its final matured version of [4], is in fact a nullspace method, however without the least order synthesis capability.

The nullspace method described in Section II is a powerful approach to solve the FDP in the most general setting. The method is applicable to both standard and descriptor systems, in both continuous- and discrete-time. This approach can relatively easily handle the least order synthesis problem and can produce detectors with optimal sensitivity condition of residual to several faults. The underlying computational algorithms are numerically reliable, consisting of either numerically stable procedures or computational sequences for which possible lost of numerical stability can be reliably detected. Therefore, the nullspace method can be considered as being the only completely satisfactory method able to serve as basis for robust numerical software implementation. Such software is available in a recently developed FAULT Detection Toolbox for Matlab [33], [34].

\section{REFERENCES}

[1] X. Ding and P. M. Frank, "Frequency domain approach and threshold selector for robust model-based fault detection and isolation," Proc. of IFAC Symposium SAFEPROCESS 1991, Baden-Baden, Germany, 1991.

[2] M. Nyberg, "Criterions for detectability and strong detectability of faults in linear systems," Int. J. Control, vol. 75, pp. 490-501, 2002.

[3] R. J. Patton and M. Hou, "Design of fault detection and isolation observers: a matrix pencil approach," Automatica, vol. 34, no. 9, pp. 1135-1140, 1998.

[4] M. Hou, "Fault detection and isolation for descriptor systems," in Issues of Fault Dyagnosis for Dynamic Systems, R. J. Patton, P. M. Frank, and R. N. Clark, Eds. Springer Verlag, London, 2000, pp. $115-144$.

[5] J.-F. Magni and P. Mouyon, "On residual generation by observer and parity space approaches," IEEE Trans. Automat. Control, vol. 39, pp. $441-447,1994$.

[6] J. Gertler, "All linear methods are equal - and extendible to (some) nonlinearities," International Journal of Robust and Nonlinear Control, vol. 12, pp. 629-648, 2002.

[7] E. Frisk and M. Nyberg, "A minimal polynomial basis solution to residual generation for fault diagnosis in linear systems," Automatica, vol. 37, pp. 1417-1424, 2001.
[8] M. Nyberg and E. Frisk, "Residual generation for fault diagnosis of systems described by linear differential-algebraic equations," IEEE Trans. Automat. Control, vol. 51, pp. 1995-2000, 2006.

[9] A. Varga, "On computing least order fault detectors using rational nullspace bases," Proc. of IFAC Symp. SAFEPROCESS'2003, Washington D.C., 2003.

[10] _ _ "On designing least order residual generators for fault detection and isolation," Proc. 16th Internat. Conf. on Control Systems and Computer Science, Bucharest, Romania, 2007, pp. 323-330.

[11] _ , "On computing nullspace bases - a fault detection perspective," in Proc. IFAC 2008 World Congress, Seoul, Korea., 2008, pp. 62956300.

[12] T. Beelen, "New algorithms for computing the kronecker structure of a pencil with applications to systems and control theory," Ph. D. Thesis, Eindhoven University of Technology, 1987.

[13] A. Varga, "Computation of Kronecker-like forms of a system pencil: Applications, algorithms and software," Proc. CACSD'96 Symposium, Dearborn, MI, 1996, pp. 77-82.

[14] _ _ "Reliable algorithms for computing minimal dynamic covers for descriptor systems," Proc. of MTNS'04, Leuven, Belgium, 2004.

[15] J. Gertler, Fault Detection and Diagnosis in Engineering Systems. New York: Marcel Dekker, 1998.

[16] K. Zhou, J. C. Doyle, and K. Glover, Robust and Optimal Control. Prentice Hall, 1996.

[17] A. Varga, "Computation of coprime factorizations of rational matrices," Lin. Alg. \& Appl., vol. 271, pp. 83-115, 1998.

[18] E. Y. Chow and A. S. Willsky, "Analytical redundancy and the design of robust failure detection systems," IEEE Trans. Automat. Control, vol. 29 , pp. $603-614,1984$.

[19] X.-C. Lou, A. S. Willsky, and G. C. Verghese, "Optimally robust redundancy relations for failure detection in uncertain systems," $\mathrm{Au}$ tomatica, vol. 22, pp. 333-344, 1986.

[20] D. Maquin, B. Gaddouna, and J. Ragot, "Generation of parity equations for singular systems. application to diagnosis," Proc. of Int. Conference on Systems, Man and Cybernetics, Le Touquet, France, vol. 3, 1993, pp. 400-405.

[21] P. M. Frank, S. X. Ding, and T. Marcu, "Model-based fault diagnosis in technical processes," Trans. of Inst. Meas. \& Control, vol. 22, pp. 57-101, 2000.

[22] M. A. Massoumnia, "A geometric approach to failure detection and identification in linear systems," Ph.D. Dissertation, Dept. Aeronautics and Astronautics, MIT, Cambridge, MA, 1986.

[23] S. X. Ding, L. Guo, and T. Jeinsch, "A characterization of parity space and its application to robust fault detection," IEEE Trans. Automat. Control, vol. 44, pp. 337 - 343, 1999.

[24] C. C. Paige, "Properties of numerical algorithms related to computing controllability," IEEE Trans. Automat. Control, vol. 26, pp. 130 - 138, 1981.

[25] M. Hou and P. C. Müller, "Fault detection and isolation observers," Int. J. Control, vol. 60, pp. 827-846, 1994.

[26] R. V. Beard, "Failure accomodation in linear systems through selfreorganization," Ph. D. Dissertation, Dept. Aeronautics and Astronautics, Mass. Inst. Technology, Cambridge, MA, 1971.

[27] H. L. Jones, "Failure detection in linear systems," Ph. D. Dissertation, Dept. Aeronautics and Astronautics, MIT, Cambridge, MA, 1971.

[28] J. E. White and J. L. Speyer, "Detection filter design: Spectral theory and algorithms," IEEE Trans. Automat. Control, vol. 32, pp. 593-603, 1987.

[29] J. Chen and R. J. Patton, Robust Model-Based Fault Diagnosis for Dynamic Systems. Kluwer Academic Publishers, London, 1999.

[30] A. Saberi, A. A. Stoorvogel, and P. Sannuti, Filtering Theory. Birkhauser, 2007.

[31] J. Wünnenberg, "Observer-based fault detection in dynamic systems," Ph.D. Dissertation, University of Duisburg, 1990.

[32] L. M. Silverman and H. J. Payne, "Input-output structure of linear systems with application to the decoupling problem," SIAM Journal on Control, vol. 9, no. 2, pp. 199-233, 1971.

[33] A. Varga, "A fault Detection toolbox for Matlab," Proc. of CACSD'06, Munich, Germany, 2006.

[34] — "Linear FDI-Techniques and Software Tools," German Aerospace Center (DLR), Institute of Robotics and Mechatronics, FAULT DETECTION Toolbox V0.8 - Technical Documentation IB 51508-18, 2008. 